\title{
Update of the Preventive Antibiotics in Stroke Study (PASS): statistical analysis plan
}

Willeke F Westendorp ${ }^{1 \dagger}$, Jan-Dirk Vermeij ${ }^{1 \dagger}$, Diederik W J Dippel ${ }^{3}$, Marcel G W Dijkgraaf ${ }^{2}$, Tom van der Poll ${ }^{4,5}$, Jan M Prins ${ }^{4,5}$, Frederique H Vermeij ${ }^{6}$, Yvo B W E M Roos ${ }^{1}$, Matthijs C Brouwer ${ }^{1,4}$, Aeilko H Zwinderman', Diederik van de Beek ${ }^{1,4^{*}+}$ and Paul J Nederkoorn ${ }^{1+}$

\begin{abstract}
Background: Infections occur in 30\% of stroke patients and are associated with unfavorable outcomes. Preventive antibiotic therapy lowers the infection rate after stroke, but the effect of preventive antibiotic treatment on functional outcome in patients with stroke is unknown. The PASS is a multicenter, prospective, phase three, randomized, open-label, blinded end-point (PROBE) trial of preventive antibiotic therapy in acute stroke. Patients are randomly assigned to either ceftriaxone at a dose of $2 \mathrm{~g}$, given every $24 \mathrm{~h}$ intravenously for 4 days, in addition to standard stroke-unit care, or standard stroke-unit care without preventive antibiotic therapy. The aim of this study is to assess whether preventive antibiotic treatment improves functional outcome at 3 months by preventing infections. This paper presents in detail the statistical analysis plan (SAP) of the Preventive Antibiotics in Stroke Study (PASS) and was submitted while the investigators were still blinded for all outcomes.
\end{abstract}

Results: The primary outcome is the score on the modified Rankin Scale (mRS), assessed by ordinal logistic regression analysis according to a proportional odds model. Secondary analysis of the primary outcome is the score on the mRS dichotomized as a favorable outcome ( $\mathrm{mRS} 0$ to 2) versus unfavorable outcome (mRS 3 to 6). Secondary outcome measures are death rate at discharge and 3 months, infection rate during hospital admission, length of hospital admission, volume of post-stroke care, use of antibiotics during hospital stay, quality-adjusted life years and costs. Complications of treatment, serious adverse events (SAEs) and suspected unexpected serious adverse reactions (SUSARs) are reported as safety outcomes.

Conclusions: The data from PASS will establish whether preventive antibiotic therapy in acute stroke improves functional outcome by preventing infection and will be analyzed according to this pre-specified SAP.

Trial registration: Current controlled trials; ISRCTN66140176. Date of registration: 6 April 2010.

Keywords: stroke, infection, antibiotics, randomized clinical trial, statistical analysis plan

\section{Update} Introduction

Stroke is a leading cause of death worldwide [1]. Infections occur in $30 \%$ of stroke patients and are associated with unfavorable outcomes [2,3]. Preventive antibiotic therapy lowers infection rate in patients after stroke, but the effect

\footnotetext{
* Correspondence: d.vandebeek@amc.uva.nl

${ }^{\dagger}$ Equal contributors

'Department of Neurology, Academic Medical Center, P.O. Box 22660, 1100 DD Amsterdam, the Netherlands

${ }^{4}$ Department of Neurology, Center of Infection and Immunity (CINIMA), Academic Medical Center, P.O. Box 22660, 1100 DD Amsterdam, the Netherlands

Full list of author information is available at the end of the article
}

of preventive antibiotic treatment on functional outcome after stroke has not yet been investigated $[4,5]$. The Preventive Antibiotics in Stroke Study (PASS) is a phase three randomized clinical trial investigating whether the preventive use of the antibiotic ceftriaxone improves functional outcome in acute stroke patients by preventing infections. We previously published the trial protocol and an update of this protocol; we now present the statistical analysis plan (SAP) [6,7]. This SAP was drafted without knowledge of any of the outcomes by the investigators and randomization code will not be broken before acceptance of the current paper for publication. 


\section{Summary study protocol}

PASS is a multicenter prospective, randomized, phase III, open-label, blinded end-point superiority trial (PROBE) of standard care with preventive ceftriaxone treatment compared to standard care without preventive ceftriaxone. Adult patients with stroke (both ischemic and hemorrhagic), a score $\geq 1$ on the National Institutes of Health Stroke Scale (NIHSS) and stroke onset within 24 hours were included [8]. Patients were excluded in case of infection at admission, use of antibiotics within 24 hours before admission, previous hypersensitivity or anaphylaxis to cephalosporins or penicillin, subarachnoid hemorrhage, pregnancy or when death seemed imminent. Patients were randomly assigned to either ceftriaxone at a dose of $2 \mathrm{~g}$, given every $24 \mathrm{~h}$ intravenously for 4 days, in addition to stroke-unit care, or standard stroke-unit care without preventive antibiotic therapy. Randomization was performed through ALEA (online software for randomized trials; https://nl.tenalea.net/amc/ ALEA/Login.aspx) and is based on a uniform distribution; weight of the arms is equal (1:1). Randomization is stratified according to study center (academic hospital, large nonacademic hospital, or small non-academic hospital) and stroke severity (score on NIHSS 1 to 9 or $>9$ ) and performed by using random blocks with a maximum block size of 6; blocks of 2, 4 and 6 are made per stratum combination [9]. The study has a PROBE design, which implies that blinding is lost, but only as to treatment. Patient and physician were aware of treatment allocation; however, the assessors of outcome were not. Data were collected on admission, during hospital stay, and at 3 months by standardized case record forms. The primary outcome is functional outcome at 3 months follow-up, as assessed on the mRS during a structured telephone interview by a trained assessor blinded for treatment allocation. Secondary outcomes are death rate at discharge and at 3 months, infection rate during hospital admission, length of hospital admission, volume of post-stroke care, use of antibiotics during hospital stay, quality adjusted life years (QALYs) and costs. Safety outcomes are complications of treatment, Serious adverse events (SAEs) and suspected unexpected serious adverse reactions (SUSARs). In the initial trial protocol, we presented a binary logistic regression analysis on the dichotomized mRS ( 0 to 2 versus 3 to 6 ) as primary outcome, requiring a sample size of 3,200 patients, and a proportional odds model in a secondary analysis of the primary end point [9]. Blinded for any of the outcomes, we have changed the primary analysis in PASS from a binary logistic to an ordinal logistic regression on the original mRS, enhancing statistical power. The adapted power analysis showed that with identical assumptions on the clinical effect, using a 0.05 two-sided significance level and $80 \%$ study power, 2,550 patients were needed [9]. The analysis of dichotomized mRS data will now be the secondary analysis of the primary end point.

On 23 March 2014, all patients were included and the last follow-up is expected in June 2014. For the complete study protocol and update, we refer to previous publications $[6,7]$.

\section{Protocol developments}

PASS is registered at current controlled trials (www.controlled-trials.com; ISRCTN: 66140176; date of registration: 6 April 2010). The medical-ethical board of the Academic Medical Center, Amsterdam, approved the protocol on 5 May 2010, and 29 Dutch participating centers were added in the course of the study. Due to the change in primary analysis of primary outcome from a binary logistic approach to an ordinal logistic regression analysis and an expected rate of patients lost to follow-up and/or patients with incomplete data of $5 \%$, the total sample size was reduced from 3,200 patients to 2,550 patients in 2014 [7]. Importantly, no changes were made regarding the primary outcome measurement (that is, the assumed size of the effect on the mRS). This update of the protocol was recently published in this journal [7].

\section{Statistical analysis plan \\ General analysis principles}

The code of the database will not be broken until all efficacy and safety data up to the last patient are included in the database, after data verification and validation are performed, and after the SAP has been accepted for publication. Analysis will be performed by the investigators of the PASS study group (see Acknowledgements section) assisted by a biostatistician of the Academic Medical Centre in Amsterdam.

\section{Patient flow diagram}

The flow of participants will be displayed in the Consolidated Standards of Reporting Trials (CONSORT) Flow diagram (Figure 1). Due to the pragmatic design of the study, the total number patients assessed for eligibility has not been assessed.

\section{Definition of intention-to-treat and per-protocol population}

Main analysis will be performed according to the intention to treat (ITT) principle. The safety analysis will be performed in a per protocol (PP) analysis. If a patient was by fault randomized more than once, the first randomization outcome was used. Patients who withdrew consent directly after randomization (that is, before treatment was initiated in those randomized for ceftriaxone in addition to standard care, or within 6 hours after randomization in those randomized for standard care) will be excluded from analysis. Patients with protocol deviations in eligibility are 


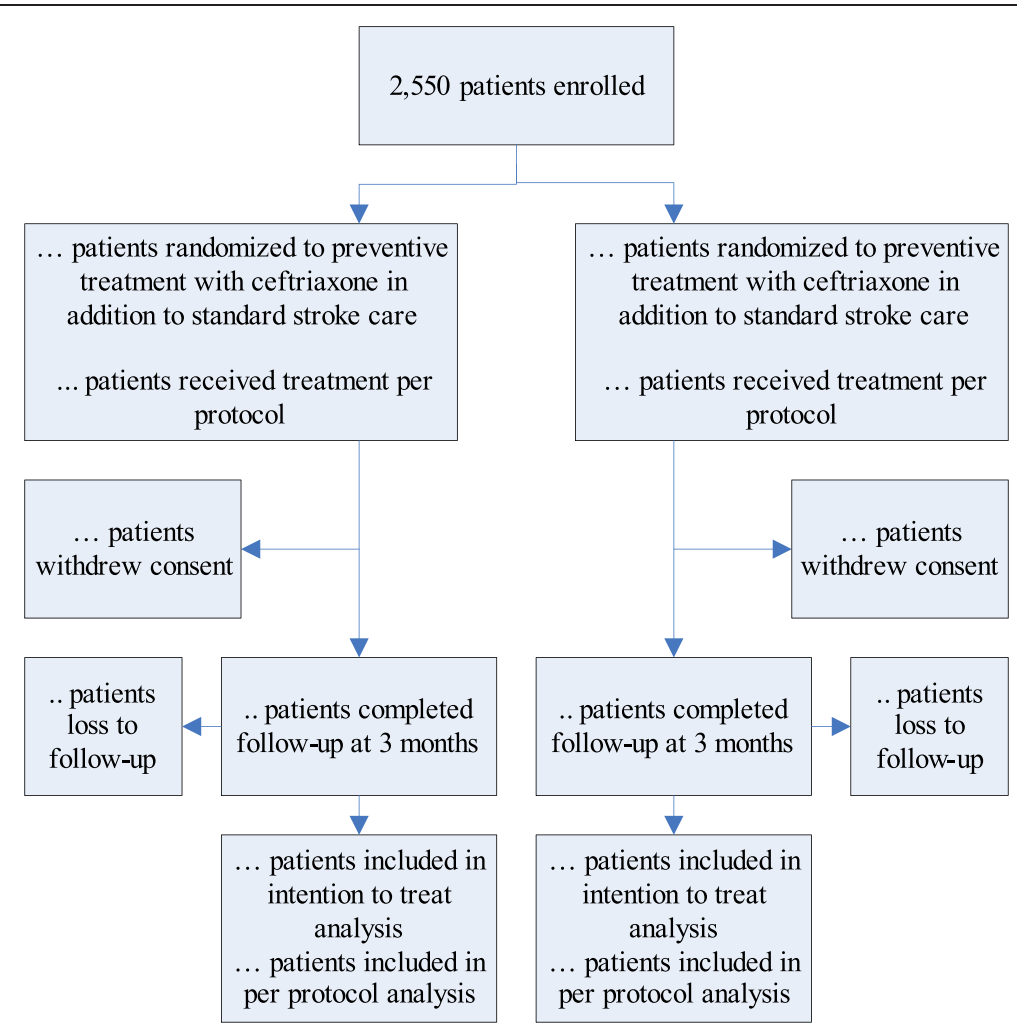

Figure 1 Flow-chart of patients.

included in the ITT analysis and will be tabulated (Table 1). Patients not receiving their allocated treatment due to instantaneous crossover are considered protocol violations; these patients will be included in the ITT population. PP analysis will exclude patients for whom protocol deviations in treatment and eligibility were made (see protocol deviations in eligibility and protocol deviations in treatment).

\section{Handling of missing data}

If outcome data could not be obtained at the 3 month evaluation, we will first check the municipal council to ensure that the patient is not deceased. All other patients are considered lost to follow-up and will be tabulated, including the percentage of missing outcome data and the association with treatment. Missing outcome data will be obtained by imputation, using the coefficients of five rounds of imputation to obtain the final estimates. We will perform sensitivity analysis. First, we will use single imputation by last observation carried forward (LOCF). An observer blinded for treatment allocation will obtain the last observational score on the mRS using the medical charts and the letters of discharge of the stroke episode. All patients with LOCF will be tabulated with an explanation for the loss to follow-up (Table 2).

We will also perform a sensitivity analysis of baseline characteristics of the group of patients not lost-to-follow-
Table 1 Number and type of protocol violations in eligibility

\begin{tabular}{lcc}
\hline $\begin{array}{l}\text { Type of protocol } \\
\text { violation in eligibility }\end{array}$ & $\begin{array}{c}\text { Ceftriaxone }+ \text { standard care } \\
(\mathbf{n}=\ldots)\end{array}$ & $\begin{array}{c}\text { Standard care } \\
(\mathbf{n}=\ldots)\end{array}$ \\
\hline
\end{tabular}

Age $<18$ years

Stroke

No neurological

symptoms (NIHSS =0)

Onset of stroke $>24$

hours ago

Admission

Infection at admission

Use of antibiotics $<24$

hours before admission

Pregnancy

Known hypersensitivity to cephalosporins

Previous anaphylaxis for penicillin derivates

Subarachnoidal

hemorrhage

Death is imminent

Total number of

protocol violations in eligibility 
Table 2 Assessment of follow-up by LOCF according to treatment allocation

\begin{tabular}{lll}
\hline Patient number & Explanation & Treatment allocation \\
\hline
\end{tabular}

up versus all patients included in PASS. In addition, we will also perform a joint model analysis of the loss to follow-up and the mRS change during follow-up [10]. Missing values of baseline characteristics will not be included or imputed in the display of baseline characteristics. When values are missing for dichotomous variables, the actual denominator will be stated. In case of continuous variables, a footnote will be added to show the number of patients for whom the variable was missing.

\section{Protocol deviations in eligibility, consent procedure, treatment}

When a patient was randomized but did not adhere to inclusion or exclusion criteria, this was considered a protocol deviation regarding eligibility. Patients with protocol deviations in eligibility were included in the ITT analysis, but excluded from PP analysis.

In each center, the local investigator obtained written informed consent from the patient or representative according to the PASS study protocol. Patients who withdrew consent directly after randomization were excluded from further analysis. The flow of patients is displayed in the CONSORT flowchart (Figure 1).

Treatment allocation was regarded as carried out according to the study protocol when a patient randomized for ceftriaxone in addition to standard care received ceftriaxone 2 gram each 24 hours for 4 days. Patients were also considered as treated PP when treatment was terminated within 4 days due to discharge, death, a palliative care policy, an allergic reaction without anaphylaxis or a previous allergic reaction in medical history (see inclusion and exclusion criteria and protocol deviation in eligibility), other side effects of treatment, or when treatment with ceftriaxone was changed into treatment with another antibiotic because of an infection because these situations and what to do were all defined and described in the initial protocol [6]. In patients allocated to standard care, treatment was carried out according to the study protocol when patients did not receive preventive antibiotic therapy.

\section{Baseline characteristics}

Baseline characteristics of all patients will be outlined per treatment allocation in a baseline table describing the following variables: age, sex, medical history (atrial fibrillation/ flutter, stroke, hypercholesterolemia, hypertension, myocardial infarction, cardiac valve insufficiency/stenosis/replacement, peripheral vascular disease, obstructive pulmonary disease, and immunocompromised), current smoking, specific medication (anticoagulants, antiplatelet, statin, ACE inhibitor, $\beta$-blocker, and proton pump inhibitor) prior to stroke, disability prior to stroke on mRS, stroke severity on NIHSS, performance of a screening test for swallowing function, dysphagia, acute treatment (IV thrombolysis and anticoagulant antagonist therapy) and diagnosis at discharge (infarct, haemorrhage, TIA, or other). Outline of the table is displayed in the 'Outline of figures and tables' section (Table 3). All variables will be presented categorized by treatment arm. Dichotomous variables will be displayed in percentage with the number of patients divided by the total number of evaluated patients. Continuous variables will be reported as means with standard deviations when normally distributed and in medians with interquartile ranges when they do not meet the criterion of being normally distributed, as assessed by the Kolmogorov-Smirnov test. For continuous variables, the number of patients evaluated will be presented in a footnote of Table 3.

\section{Assessment of primary outcome}

A structured telephone interview with each patient was held at 3 months by one of three trained research nurses, blinded for treatment allocation, to assess the primary outcome on the mRS. This structured telephone interview was validated in an earlier study [11].

\section{Assessment of secondary outcomes}

The assessment of secondary outcomes will be performed as described below, for each outcome separately:

\section{Infection rate during hospital admission}

The total number of patients diagnosed with one or more infection(s) during hospital admission will be reported, as well as the total number of infections. Infections will be reported according to subtypes pneumonia, urinary tract infection and other infection. Infection will be assessed in two ways. First, the infection will be diagnosed in the clinical setting by the treating physician and registered as pneumonia, urinary tract infection or other infection. The clinical diagnosis of infection will be used for the primary analysis. Suspected infections without diagnostics being performed are also recorded and reported as such (for example, in a patient with a palliative care policy). Second, infection will be categorized by two infectious disease specialists who are blinded for treatment allocation, using the modified criteria of the Centers for Disease Control and Prevention (CDC criteria) [12]. For this second categorization, patients with fever, new onset delirium or clinical diagnosis of infection during hospital admission will be reviewed. For this purpose, data on the diagnostic procedures during admission as recorded in the Case Record Form (CRF) will be used. For the diagnosis of pneumonia and urinary tract infection prespecified algorithms will be used based on the CDC-criteria (Figures 2 and 3). 
Table 3 Baseline characteristics

\begin{tabular}{|c|c|c|}
\hline Baseline characteristics & $\begin{array}{l}\text { Ceftriaxone + } \\
\text { standard care }\end{array}$ & $\begin{array}{l}\text { Standard } \\
\text { care }\end{array}$ \\
\hline \multicolumn{3}{|l|}{ Age - years } \\
\hline \multicolumn{3}{|l|}{ Male sex - \% n/N } \\
\hline \multicolumn{3}{|l|}{ Medical history - \% n/N } \\
\hline \multicolumn{3}{|l|}{ - Atrial fibrillation/flutter } \\
\hline \multicolumn{3}{|l|}{ - Stroke } \\
\hline \multicolumn{3}{|l|}{ - Hypercholesterolemia } \\
\hline \multicolumn{3}{|l|}{ - Hypertension } \\
\hline \multicolumn{3}{|l|}{ - Myocardial infarction } \\
\hline \multicolumn{3}{|l|}{$\begin{array}{l}\text { - Cardiac valve insufficiency/stenosis/ } \\
\text { replacement }\end{array}$} \\
\hline \multicolumn{3}{|l|}{ - Peripheral vascular disease } \\
\hline \multicolumn{3}{|l|}{ - Obstructive pulmonary disease } \\
\hline \multicolumn{3}{|l|}{ - Immunocompromised } \\
\hline \multicolumn{3}{|l|}{ Current smoker - \% n/N } \\
\hline \multicolumn{3}{|l|}{ Medication prior to stroke - $\% \mathrm{n} / \mathrm{N}$} \\
\hline \multicolumn{3}{|l|}{ - Anticoagulants } \\
\hline \multicolumn{3}{|l|}{ - Antiplatelet } \\
\hline \multicolumn{3}{|l|}{ - Statin } \\
\hline \multicolumn{3}{|l|}{ - ACE inhibitor } \\
\hline \multicolumn{3}{|l|}{ - Bèta-blocker } \\
\hline \multicolumn{3}{|l|}{ - Proton pump inhibitor } \\
\hline \multicolumn{3}{|l|}{ Disability prior to stroke - mRS * } \\
\hline \multicolumn{3}{|l|}{ Stroke severity - NIHSS ** } \\
\hline \multicolumn{3}{|l|}{$\begin{array}{l}\text { Swallowing screening performed - \% } \\
\mathrm{n} / \mathrm{N}\end{array}$} \\
\hline \multicolumn{3}{|l|}{ Dysphagic patients - \% n/N } \\
\hline \multicolumn{3}{|l|}{ Acute treatment - $\% \mathrm{n} / \mathrm{N}$} \\
\hline \multicolumn{3}{|l|}{ - IV thrombolysis } \\
\hline \multicolumn{3}{|l|}{ - Coagulant therapy } \\
\hline \multicolumn{3}{|l|}{ Diagnosis at discharge - $\% \mathrm{n} / \mathrm{N}$} \\
\hline \multicolumn{3}{|l|}{ - Infarction } \\
\hline \multicolumn{3}{|l|}{ - Hemorrhage } \\
\hline \multicolumn{3}{|l|}{ - Transient ischemic attack (TIA) } \\
\hline - Other & & \\
\hline
\end{tabular}

**NIHSS denotes National Institutes of Health Stroke Scale.

Patients with a positive blood culture or a positive culture from the presumed site of infection, other than the lungs or urine, with a clinically relevant pathogen will be diagnosed as 'other infection.' Patients will be categorized as having confirmed pneumonia, urinary tract infection, or other infection. Only bacterial infections will be assessed since preventive antibiotic therapy aims to reduce these infections. Infection with Clostridium difficile is reported as a treatment complication. Case definition of this infection is diarrhea plus a positive $C$. difficile toxin test. Clostridium infection was diagnosed by the treating physician and was reviewed by the expert panel.

\section{Death rate at discharge and at 3 months}

Death during hospital admission was recorded in the CRF by the treating physician and notified as an SAE to the trial office. Death was also registered at the 3 months follow-up. If needed, survival status at 3 months was evaluated through contact with general practitioners and the municipality register.

\section{Length of hospital stay}

The day of admission and discharge was recorded in the CRF by the treating physician. Length of hospital admission is measured in days.

\section{Total use of antibiotics during hospital stay}

The use of antibiotics other than preventive antibiotic therapy will be recorded in the case record form. Total antibiotic use will be recorded in units of the 'defined daily dose' (DDD) and the number of days of use. For definitions of the DDD, classification according to the World Health Organization (WHO) will be used for each antibiotic [13].

\section{Volume of post-stroke care, cost-effectiveness analysis}

The cost-effectiveness will be measured by an economic analysis conducted alongside the study. This analysis is not included in the publication to which this analysis plan applies.

\section{Assessment of safety outcomes}

Safety outcomes are complications of treatment, SAEs and SUSARs. All SAEs and SUSARs during the hospital stay are recorded in case record forms by the treating physician and reported to the trial office. SAEs and SUSARs occurring after discharge are recorded during the follow-up interview at 3 months. The physician records treatment complications in the CRF (diarrhea caused by C. difficile, allergic reaction that caused cessation of ceftriaxone, infection with ceftriaxone resistant microorganism, phlebitis at place of IV-catheter, elevation of liver enzymes, oliguria or elevation of serum creatinine).

Cause of death will be reviewed by two independent observers. They will use information from the hospital discharge letter or the medical correspondence received by the general practitioner in case the patient died after discharge. Discrepancies will be reviewed in a consensus meeting in the presence of a third investigator. Outcome parameters were derived from three recent cardiovascular trials and were modified for expected outcomes in our study [14-16]. A distinction will be made among a cardiovascular cause (brain infarction, brain hemorrhage, 


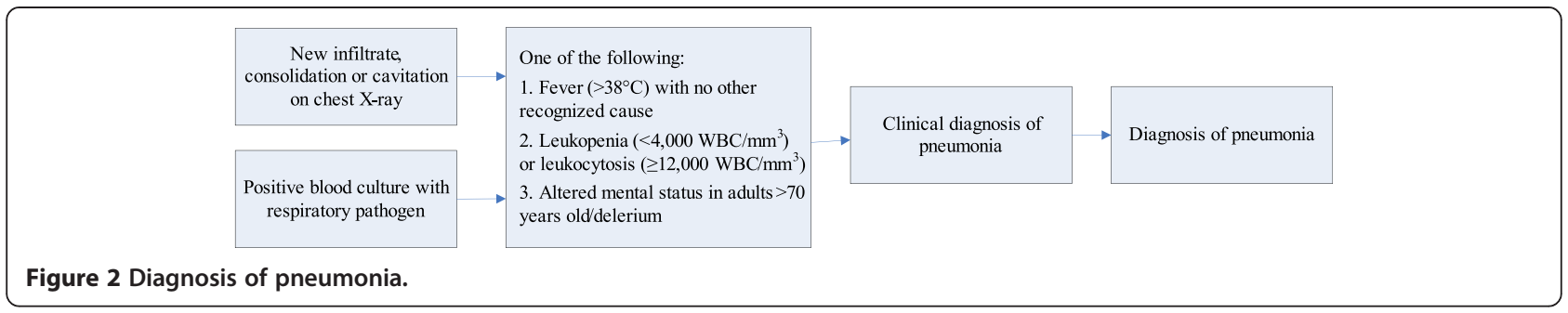

myocardial- or pulmonary embolism. or another cardiovascular cause), an infection (pneumonia, sepsis or another infection), death by any type of malignancy, death by any other cause (for example, traffic accident), withdrawal of treatment due to a poor prognosis or unknown cause of death.

\section{Analysis of primary outcome}

An ordinal regression model on the total range of the mRS will be performed as the first analysis of primary outcome, under the assumption of proportional odds [7]. The distribution of primary outcome (for example, functional outcome on the mRS) in both treatment groups will be expressed in a histogram (Figure 4). Both adjusted and unadjusted analyses will be performed and reported. In clinical trials, adjusting for prognostic covariates improves statistical power, can correct for imbalances in baseline prognostic variables and can reduce variability in data $[17,18]$. The choice of prognostic covariates is mostly based on imbalances across treatment groups, prognostic factors that are related to the primary outcome, or a combination of both [17]. As the investigators are blinded for all outcome data until the statistical analysis plan is accepted for publication, we chose to use the most important prognostic factors for outcome after stroke: age, stroke severity on the NIHSS, history of stroke, history of diabetes, prior disability as defined on $\mathrm{mRS}$, and stroke type [19]. Stratification of randomization was performed according to both study center and stroke severity, so we will also include study center as a covariate. The second analysis of the primary endpoint, that is, the dichotomized score on the mRS (for example, favorable versus unfavorable, mRS 0 to 2 versus mRS 3 to 6), will be expressed as OR with 95\% confidence intervals (CI; Table 4). Results of the dichotomized approach will be compared to the results of the primary analysis of primary outcome.

\section{Analysis of secondary outcomes}

The number of patients with one or more in-hospital poststroke infection(s) will be presented as numbers with event of numbers evaluated and analyzed using the chi-square test, and OR estimates with $95 \% \mathrm{CI}$. Infection rates will be reported as 'judged by treating physicians' and 'infectious diseases panel.' Death rate at discharge and at 3 months will also be analyzed using the chi-squared test and presented as OR estimates and 95\% CI. Use of antibiotics in defined daily doses and length of hospital admission will be analyzed using the two group t-test or Mann- Whitney test where appropriate (Table 4). The analysis of volume of post-stroke care, use of antibiotics during 3 months followup and the cost-effectiveness analysis will be analyzed using a separate analysis protocol and presented in a subsequent paper and is, therefore, not discussed here.

\section{Safety outcomes}

Complications of treatment, SAE's and SUSAR's per patient will be tabulated according to treatment group, and analyzed using the chi-squared test (Table 5 and table 6).

\section{Subgroup analysis of primary and secondary outcomes}

We will perform the following sub-group analyses for the primary outcome: stroke type (infarction or hemorrhage), stroke severity (NIHSS 1 to 9 or NIHSS 10 to 30), time between stroke symptoms and start of the antibiotic treatment ( 0 to $12 \mathrm{~h}$ versus 12 to $24 \mathrm{~h}$ ) and age. For the subgroup analysis of primary analysis of primary outcome, the single OR from the proportional odds model will be

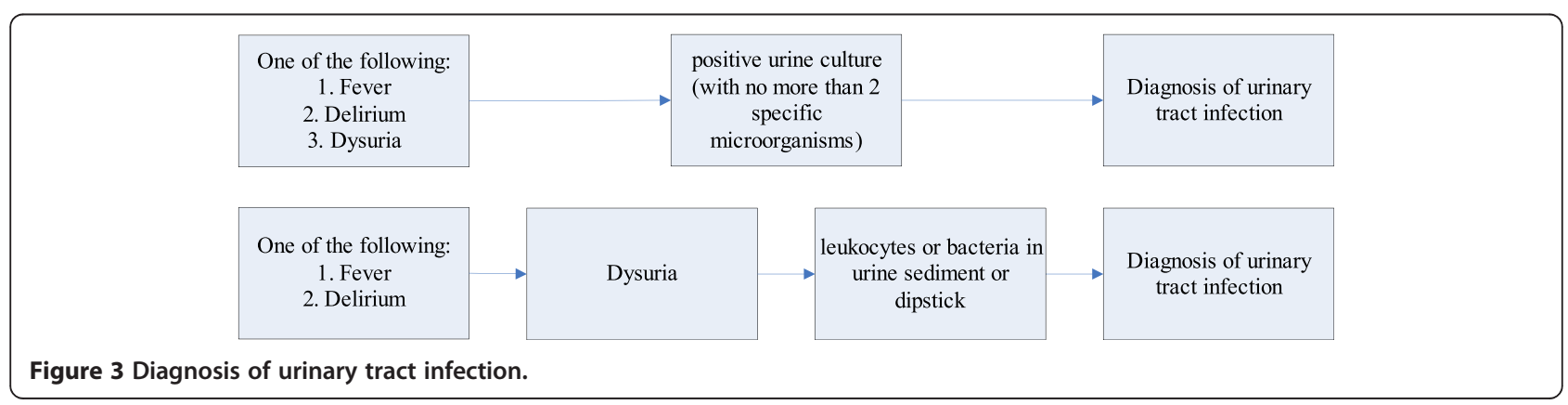




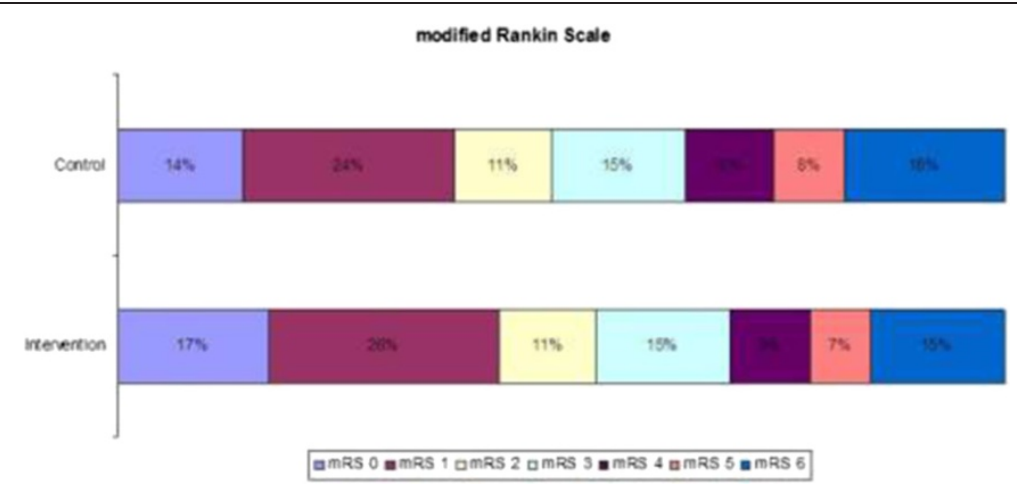

Figure 4 Graphic display of primary outcome.

calculated for each subgroup separately. For the subgroup analysis of secondary analysis of primary outcome, we will tabulate the results and analyze them using the chisquared test and presented as OR and 95\% CI ((Table 7: subgroup analysis of primary outcome). In addition to these predefined subgroup analyses, we will perform a larger set of exploratory additional analyses. For secondary outcomes, we will perform all the previous mentioned subgroup analyses (stroke type, severity, time to treatment, and age). In addition we will perform analysis on presence of a swallowing disorder, respiratory tract infections, and a urinary catheter.

\section{Authorships}

Two PhD students (WFW and J-DV) of this project will share first authorship; the two principle investigators (PIs) of this project will share last authorship (DvdB and PJN;
DvdB corresponding author); local investigators who included at least 100 patients will be co-author; PASS study group members and physicians in expert panels for outcome-scoring will be co-author; and all local investigators who included less than 100 patients in PASS will be explicitly listed in the PASS investigators list.

\section{Discussion}

The aim of our study is to investigate whether preventive antibiotic therapy improves functional outcome by reducing the number of infections in acute stroke patients. With this SAP, we present the analyses that will be published in the primary publication. By publishing the statistical analysis plan before knowledge of any outcome, we stimulate transparency of scientific conduct and allow others to add timely suggestions for additional analyses.

\section{Table 4 Secondary outcomes}

$\begin{array}{llll}\text { Ceftriaxone }+ \text { standard care }(\mathrm{n}=\ldots) & \text { Standard care }(\mathrm{n}=\ldots) & P & \text { OR } 95 \% \mathrm{Cl}\end{array}$

Secondary analysis of primary outcome

Favorable outcome - \% n/N

Secondary outcomes:

Clinical diagnosis of infection during admission - $\mathrm{n}$

- Pneumonia

- Urinary tract infection

- Other

Diagnosis of infection based on expert panel - $n$

- Pneumonia

- Urinary tract infection

- Other

Mortality - \% n/N

- At discharge

- At 3 months

Length of hospital stay - days 
Table 5 Number and type of serious adverse events

\begin{tabular}{|c|c|c|}
\hline $\begin{array}{l}\text { Type of SAE* }-\% \\
\mathrm{n} / \mathrm{N}\end{array}$ & $\begin{array}{l}\text { Ceftriaxone + standard care } \\
(\mathrm{n}=\ldots)\end{array}$ & $\begin{array}{c}\text { Standard care } P \\
(\mathrm{n}=\ldots)\end{array}$ \\
\hline \multicolumn{3}{|l|}{ - Death } \\
\hline \multicolumn{3}{|l|}{$\begin{array}{l}\text { - Life-threatening } \\
\text { event }\end{array}$} \\
\hline \multicolumn{3}{|l|}{ - New hospitalization } \\
\hline \multicolumn{3}{|l|}{$\begin{array}{l}\text { - Prolongation of } \\
\text { existing } \\
\text { hospitalization }\end{array}$} \\
\hline \multicolumn{3}{|l|}{$\begin{array}{l}\text { - Persistence of } \\
\text { significant disability } \\
\text { or incapacity }\end{array}$} \\
\hline $\begin{array}{l}\text { Total number of } \\
\text { SAE's }\end{array}$ & & \\
\hline
\end{tabular}

*SAE, serious adverse event.

Patients in the acute phase of stroke are at risk for infections. A systematic review and meta-analysis of 87 studies showed that infections complicate stroke in 30\% of all stroke patients. Pneumonia was associated with mortality with an OR of 3.62 (95\% CI 2.80 to 4.68) [3]. The effect of preventive antibiotic therapy on outcome in stroke patients has been investigated in few studies. Two meta-analyses of these studies showed that preventive antibiotic therapy reduced the number of infections $[4,5]$. The proportion of patients who died and the number of disabled patients were not significantly reduced, but numbers of included patients were small.

The PROBE design with open-label preventive antibiotics might introduce detection bias for infection. Physicians are aware of the treatment allocation, which potentially influences decisions on nonscheduled treatment (that is, the detection and treatment of patients with infection). This might influence the outcome measure of infection rate. To control for this bias, we will provide a secondary judgement

\section{Table 6 Complications of treatment}

\begin{tabular}{|c|c|c|}
\hline $\begin{array}{l}\text { Adverse } \\
\text { reaction - \% n/N }\end{array}$ & $\begin{array}{l}\text { Ceftriaxone }+ \text { standard care } \\
(\mathrm{n}=\ldots)\end{array}$ & $\begin{array}{c}\text { Standard care } P \\
(\mathrm{n}=\ldots)\end{array}$ \\
\hline $\begin{array}{l}\text { - Diarrhea caused by } \\
\text { C. difficile }\end{array}$ & & \\
\hline $\begin{array}{l}\text { - Allergic reaction } \\
\text { that caused } \\
\text { cessation of } \\
\text { ceftriaxone }\end{array}$ & & \\
\hline $\begin{array}{l}\text { - Infection with } \\
\text { ceftriaxone-resistant } \\
\text { microorganism }\end{array}$ & & \\
\hline $\begin{array}{l}\text { - Phlebitis at place of } \\
\text { IV-catheter }\end{array}$ & & \\
\hline $\begin{array}{l}\text { - Elevation of liver } \\
\text { enzymes }\end{array}$ & & \\
\hline $\begin{array}{l}\text { - Oliguria or } \\
\text { elevation of serum } \\
\text { creatinine }\end{array}$ & & \\
\hline
\end{tabular}

Total number of adverse reactions - number $\%$.
Table 7 Subgroup analysis of primary outcome

\begin{tabular}{|c|c|c|c|}
\hline & $\begin{array}{c}\text { Ceftriaxone }+ \text { standard } \\
\text { care }(\mathrm{n}=\ldots)\end{array}$ & $\begin{array}{c}\text { Standard care } \\
(\mathrm{n}=\ldots)\end{array}$ & $\begin{array}{cc}P & \text { OR } \\
& 95 \% \mathrm{Cl}\end{array}$ \\
\hline \multicolumn{4}{|l|}{$\begin{array}{l}\text { Favorable } \\
\text { outcome (mRS* } \\
0 \text { to } 2)-\% \mathrm{n} / \mathrm{N}\end{array}$} \\
\hline \multicolumn{4}{|l|}{ - Ischemic stroke } \\
\hline \multicolumn{4}{|l|}{$\begin{array}{l}\text { - Hemorrhagic } \\
\text { stroke }\end{array}$} \\
\hline \multicolumn{4}{|l|}{$\begin{array}{l}\text { - Transient } \\
\text { ischemic attack } \\
\text { (TIA) }\end{array}$} \\
\hline \multicolumn{4}{|l|}{ - Other } \\
\hline \multicolumn{4}{|l|}{$\begin{array}{l}\text { Favorable } \\
\text { outcome (mRS } 0 \\
\text { to } 2 \text { ) - } \% \mathrm{n} / \mathrm{N}\end{array}$} \\
\hline \multicolumn{4}{|l|}{ - NIHSS** 1 to 9} \\
\hline \multicolumn{4}{|l|}{ - NIHSS 10 to 30} \\
\hline \multicolumn{4}{|l|}{$\begin{array}{l}\text { Favorable } \\
\text { outcome (mRS } 0 \\
\text { to } 2)-\% \mathrm{n} / \mathrm{N}\end{array}$} \\
\hline \multicolumn{4}{|l|}{$\begin{array}{l}\text { - time to } \\
\text { treatment } 0 \text { to } \\
6 \mathrm{~h}\end{array}$} \\
\hline \multicolumn{4}{|l|}{$\begin{array}{l}\text { - time to } \\
\text { treatment } 6 \text { to } \\
12 \mathrm{~h}\end{array}$} \\
\hline $\begin{array}{l}\text { - time to } \\
\text { treatment } 12 \\
\text { to } 24 \mathrm{~h}\end{array}$ & & & \\
\hline
\end{tabular}

${ }^{*} \mathrm{mRS}$, denotes modified Rankin Scale.

**NIHSS denotes National Institutes of Health Stroke Scale.

of infection diagnosis by a blinded expert panel, according to $\mathrm{CDC}$ criteria. The $\mathrm{CDC}$ criteria are restrictive and use ancillary investigations such as blood tests, chest X-rays and culture results to confirm the diagnosis of infection. In clinical practice, for a stroke patient with fever, a cough and abnormalities on auscultation, a physician will often not wait for culture results or refrain from treating pneumonia when a chest X-ray does not (yet) show a consolidation.

Preventive treatment with ceftriaxone after stroke might improve outcome by preventing infections. A potential beneficial effect on functional outcome might be caused by a direct effect of prevention of infections in patients after stroke, most commonly pneumonia, but also by the result of decreased length of stay on the stroke unit of even in the hospital. A recent study of individual patient data in a meta-analysis of randomized trials of ventilatorassociated pneumonia prevention showed that an overall attributable mortality of ventilator-associated pneumonia is $13 \%$, which was mainly caused by prolonged exposure to the risk of dying due to increased length of ICU stay [20]. Ceftriaxone also has neuroprotective properties, at least in animal studies of stroke, which may be mediated 
by increased expression and activity of the glutamate transporter [21].

Antibiotics may induce overgrowth of antibiotic resistant pathogens in individual patients [22]. In the general population, selective antibiotic pressure is an important determinant of emergence and dissemination of antibiotic resistance [23,24]. Previous clinical trials on preventive antibiotic therapy in stroke, antibiotic resistance patterns of bacteria cultured from patients with or without preventive antibiotics were similar, but numbers of patients were low [25]. Previous work has showed that implementation of preventive antibiotics in the ICU did not increase resistance rates in an environment with low levels of antibiotic resistance [26]. We will compare total antibiotic use in both treatment groups during hospital stay and collect stool specimens in a nested case control study that includes 300 patients.

During the course of the study we changed the analysis of primary outcome on the mRS from a dichotomized analysis toward an ordinal regression analysis. The ordinal regression analysis is increasingly used in stroke trials because of its higher efficiency [27]. Importantly, our primary outcome (for example, functional outcome on the $\mathrm{mRS}$ ) was not changed, and the assumptions used in the initial sample size calculation were maintained. By using ordinal regression analysis, the total sample size was lowered from 3,200 patients to 2,550 patients. Using this method enables us to reduce the number of patients without changing the assumptions on the magnitude of the effect on the primary outcome scale from the original sample size calculation.

\footnotetext{
Abbreviations

CRF: Case Record Form; CONSORT: Consolidated Standards of Reporting Trials; DDD: defined daily dose'; ITT: intention to treat; LOCF: last observation carried forward; mRS: modified Rankin Scale; NIHSS: National Institutes of Health Stroke Scale; OR: odds ratio; PASS: Preventive Antibiotics in Stroke Study; PP: per protocol; QALYS: quality adjusted life years; SAE: serious adverse event; SAP: statistical analysis plan; SUSAR: suspected unexpected serious adverse reaction; TIA: transient ischemic attack;

VAP: ventilator-associated pneumonia.
}

\section{Competing interests}

The authors declare that they have no competing interests.

\begin{abstract}
Authors' contributions
WFW, JDV performed conception and design of the work and drafted the work. PJN and DvdB performed conception and design of the work and revised it critically for important intellectual content. DWJD, MGWD, TvdP, JMP, FHV, YBWEMR, MCB, AHZ were involved in design of the work, and revised it critically for intellectual content. All authors approved the final version.
\end{abstract}

\section{Acknowledgements}

The PASS study was funded by the Academic Medical Centre (AMC), by the Netherlands Organisation for Health Research and Development (ZonMW 171002302) and the Netherlands Heart Foundation (Hartstichting; 2009B095). Principal investigators of the PASS are Dr. PJ Nederkoorn and Professor D van de Beek. DvdB is supported by grants from the European Research Council (ERC Starting Grant (Proposal/Contract number 281156)), Netherlands Organization for Health Research and Development (ZonMw; NWO-Vidi grant
2010 (Proposal/Contract number 016.116.358)). The study group comprises Professor DWJ Dippel; Dr. MGW Dijkgraaf; Professor JM Prins; Dr. L Spanjaard; Professor T van der Poll; Dr. FH Vermeij. Two PhD students working on the PASS are WF Westendorp and J-D Vermeij. Trial manager and nurses are IJ Hooijenga, AG de Jong and I Stijnman- Moerman. Thirty Dutch hospitals participate in the PASS; all centers with local investigators are shown in Table. The Data Safety Monitoring Committee (DSMB) is formed by: GJ Hankey, MD, PhD, Consultant Neurologist, Head of Stroke Unit, Department of Neurology, Royal Perth Hospital, Australia (chair); A Algra, MD, PhD, Clinical Epidemiologist, Julius Centre and Department of Neurology, UMC Utrecht, the Netherlands; MJM Bonten, MD, PhD, Microbiologist, Department of Medical Microbiology and Julius Centre, University Medical Centre Utrecht, Utrecht, the Netherlands. Advisory Board of the PASS consists of Professor M Vermeulen, Department of Neurology, AMC Amsterdam, and Professor RJ de Haan, Clinical Research Unit, AMC Amsterdam.

\section{Author details}

${ }^{1}$ Department of Neurology, Academic Medical Center, P.O. Box 22660, 1100 DD Amsterdam, the Netherlands. ${ }^{2}$ Clinical Research Unit (CRU), Academic Medical Center, P.O. Box 22660, 1100 DD Amsterdam, the Netherlands. ${ }^{3}$ Department of Neurology, Erasmus MC University Medical Center, P.O. Box Postbus 2040, 3000 CA Rotterdam, the Netherlands. ${ }^{4}$ Department of Neurology, Center of Infection and Immunity (CINIMA), Academic Medical Center, P.O. Box 22660, 1100 DD Amsterdam, the Netherlands. ${ }^{5}$ Department of Infectious Diseases, Academic Medical Center, P.O Box 22660, 1100 DD Amsterdam, the Netherlands. ${ }^{6}$ Department of Neurology, Sint Franciscus Gasthuis, P.O. Box 10900, 3004 BA Rotterdam, the Netherlands. 'DDepartment of Clinical Epidemiology Biostatistics and Bioinformatics, Academic Medical Center, P.O. Box 22660, 1100 DD Amsterdam, the Netherlands.

Received: 25 June 2014 Accepted: 19 September 2014

Published: 1 October 2014

\section{References}

1. Feigin $\mathrm{VL}$, Forouzanfar MH, Krishnamurthi R, Mensah GA, Connor M, Bennett DA, Moran AE, Sacco RL, Anderson L, Truelsen T, O'Donnell M, Venketasubramanian N, Barker-Collo S, Lawes CM, Wang W, Shinohara Y, Witt E, Ezzati M, Naghavi M, Murray C, Global Burden of Diseases, Injuries, and Risk Factors Study 2010 (GBD 2010) and the GBD Stroke Experts Group: Global and regional burden of stroke during 1990-2010: findings from the Global Burden of Disease Study 2010. Lancet 2014, 383:245-254.

2. Vermeij FH, op Reimer WJ S, de Man P, van Oostenbrugge RJ, Franke CL, de Jong G, de Kort PL, Dippel DW, Netherlands Stroke Survey Investigators: Stroke-associated infection is an independent risk factor for poor outcome after acute ischemic stroke: data from the Netherlands Stroke Survey. Cerebrovasc Dis 2009, 27:465-471.

3. Westendorp WF, Nederkoorn PJ, Vermeij JD, Dijkgraaf MG, van de Beek D: Post-stroke infection: A systematic review and meta-analysis. BMC Neurol 2011, 11:110.

4. van de Beek D, Wijdicks EF, Vermeij FH, de Haan RJ, Prins JM, Spanjaard L, Dippel DW, Nederkoorn PJ: Preventive antibiotics for infections in acute stroke: a systematic review and meta-analysis. Arch Neurol 2009, 66:1076-1081.

5. Westendorp WF, Vermeij JD, Vermeij F, Den Hertog HM, Dippel DW, van de Beek D, Nederkoorn PJ: Antibiotic therapy for preventing infections in patients with acute stroke. Cochrane Database Syst Rev 2012, 1, CD008530.

6. Westendorp WF, Vermeij JD, Vermeij F, Den Hertog HM, Dippel DW, van de Beek D, Nederkoorn PJ: Preventive antibiotics in stroke study: rationale and protocol for a randomised trial. Int J Stroke 2011, 6:159-163.

7. Westendorp WF, Vermeij JD, van Geloven N, Dippel DW, Dijkgraaf MG, van der Poll T, Prins JM, Spanjaard L, Vermeij FH, Nederkoorn PJ, van de Beek D: Update on the Preventive Antibiotics in Stroke Study (PASS): a randomised controlled phase 3 clinical trial. Trials 2014, 15:133.

8. National Institute of Health: National Institute of Neurological Disorders and Stroke. Stroke Scale. 2014.

9. ALEA Software for randomisation in clinical trials. In ALEA Version-Release: 2.2 build: 2070

10. Rizopoulos D, Lesaffre E: Introduction to the special issue on joint modelling techniques. Stat Methods Med Res 2014, 23:3-10.

11. Janssen PM, Visser NA, Dorhout Mees SM, Klijn CJ, Algra A, Rinkel GJ: Comparison of telephone and face-to-face assessment of the modified Rankin Scale. Cerebrovasc Dis 2010, 29:137-139. 
12. Horan TC, Andrus M, Dudeck MA: CDC/NHSN surveillance definition of health care-associated infection and criteria for specific types of infections in the acute care setting. Am J Infect Control 2008, 36:309-332.

13. World Health Organization: ATC/DDD Index. 2014. http://www.whocc.no/ atc_ddd_index/, last updated 2013-12-19.

14. Connolly SJ, Ezekowitz MD, Yusuf S, Eikelboom J, Oldgren J, Parekh A, Pogue J, Reilly PA, Themeles E, Varrone J, Wang S, Alings M, Xavier D, Zhu J, Diaz R, Lewis BS, Darius H, Diener HC, Joyner CD, Wallentin L, RE-LY Steering Committee and Investigators: Dabigatran versus warfarin in patients with atrial fibrillation. N Engl J Med 2009, 361:1139-1151.

15. Granger CB, Alexander JH, McMurray JJ, Lopes RD, Hylek EM, Hanna M, Al-Khalidi HR, Ansell J, Atar D, Avezum A, Bahit MC, Diaz R, Easton JD, Ezekowitz JA, Flaker G, Garcia D, Geraldes M, Gersh BJ, Golitsyn S, Goto S, Hermosillo AG, Hohnloser SH, Horowitz J, Mohan P, Jansky P, Lewis BS, Lopez-Sendon JL, Pais P, Parkhomenko A, Verheugt FW, et al: Apixaban versus warfarin in patients with atrial fibrillation. N Engl J Med 2011, 365:981-992.

16. Granger CB, Alexander JH, McMurray JJ, Lopes RD, Hylek EM, Hanna M, Al-Khalidi HR, Ansell J, Atar D, Avezum A, Bahit MC, Diaz R, Easton JD, Ezekowitz JA, Flaker G, Garcia D, Geraldes M, Gersh BJ, Golitsyn S, Goto S, Hermosillo AG, Hohnloser SH, Horowitz J, Mohan P, Jansky P, Lewis BS, Lopez-Sendon JL, Pais P, Parkhomenko A, Verheugt FW, et al: Rivaroxaban versus warfarin in nonvalvular atrial fibrillation. N Engl J Med 2011, 365:883-891.

17. Gray LJ, Bath PM, Collier T: Should stroke trials adjust functional outcome for baseline prognostic factors? Stroke 2009, 40:888-894.

18. Kahan $\mathrm{BC}$, Jairath $\mathrm{V}$, Dore $\mathrm{CJ}$, Morris TP: The risks and rewards of covariate adjustment in randomized trials: an assessment of 12 outcomes from 8 studies. Trials 2014, 15:139

19. Johnston KC, Connors AF Jr, Wagner DP, Knaus WA, Wang X, Haley EC Jr: A predictive risk model for outcomes of ischemic stroke. Stroke 2000, 31:448-455.

20. Melsen WG, Rovers MM, Groenwold RH, Bergmans DC, Camus C, Bauer TT, Hanisch EW, Klarin B, Koeman M, Krueger WA, Lacherade JC, Lorente L, Memish ZA, Morrow LE, Nardi G, van Nieuwenhoven CA, O'Keefe GE, Nakos G, Scannapieco FA, Seguin P, Staudinger T, Topeli A, Ferrer M, Bonten MJ: Attributable mortality of ventilator-associated pneumonia: a meta-analysis of individual patient data from randomised prevention studies. Lancet Infect Dis 2013, 13:665-671.

21. Thöne-Reineke C, Neumann C, Namsolleck P, Schmerbach K, Krikov M, Schefe JH, Lucht K, Hörtnagl H, Godes M, Müller S, Rumschüssel K, Funke-Kaiser $H$, Villringer A, Steckelings UM, Unger T: The beta-lactam antibiotic, ceftriaxone, dramatically improves survival, increases glutamate uptake and induces neurotrophins in stroke. $J$ Hypertens 2008, 26:2426-2435

22. Hawkey PM: The growing burden of antimicrobial resistance. J Antimicrob Chemother 2008, 62(Suppl 1):i1-i9.

23. Baquero F, Negri MC, Morosini MI, Blazquez J: Antibiotic-selective environments. Clin Infect Dis 1998, 27(Suppl 1):S5-S11.

24. Schechner V, Temkin E, Harbarth S, Carmeli Y, Schwaber MJ: Epidemiological interpretation of studies examining the effect of antibiotic usage on resistance. Clin Microbiol Rev 2013, 26:289-307.

25. Harms H, Prass K, Meisel C, Klehmet J, Rogge W, Drenckhahn C, Göhler J, Bereswill S, Göbel U, Wernecke KD, Wolf T, Arnold G, Halle E, Volk HD, Dirnagl U, Meisel A: Preventive antibacterial therapy in acute ischemic stroke: a randomized controlled trial. PLOS ONE 2008, 3:e2158.

26. de Smet AM, Kluytmans JA, Blok HE, Mascini EM, Benus RF, Bernards AT, Kuijper EJ, Leverstein-van Hall MA, Jansz AR, de Jongh BM, van Asselt GJ,

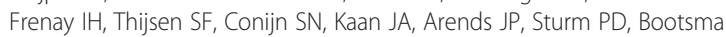
MC, Bonten MJ: Selective digestive tract decontamination and selective oropharyngeal decontamination and antibiotic resistance in patients in intensive-care units: an open-label, clustered group-randomised, crossover study. Lancet Infect Dis 2011, 11:372-380.
27. McHugh GS, Butcher I, Steyerberg EW, Marmarou A, Lu J, Lingsma HF, Weir J, Maas Al, Murray GD: A simulation study evaluating approaches to the analysis of ordinal outcome data in randomized controlled trials in traumatic brain injury: results from the IMPACT Project. Clin Trials 2010, 7:44-57.

\section{doi:10.1186/1745-6215-15-382}

Cite this article as: Westendorp et al: Update of the Preventive Antibiotics in Stroke Study (PASS): statistical analysis plan. Trials 2014 15:382

\section{Submit your next manuscript to BioMed Central and take full advantage of:}

- Convenient online submission

- Thorough peer review

- No space constraints or color figure charges

- Immediate publication on acceptance

- Inclusion in PubMed, CAS, Scopus and Google Scholar

- Research which is freely available for redistribution
C Biomed Central 